\title{
Minor sperm abnormalities in young male post-pubertal patients with juvenile dermatomyositis
}

\author{
A.J.P. Moraes ${ }^{1}$, R.M.R. Pereira ${ }^{2}$, M. Cocuzza ${ }^{3}$, R. Casemiro ${ }^{1}$, O. Saito ${ }^{4}$ and \\ C.A.A. Silva ${ }^{1}$
}

1 Unidade de Reumatologia Pediátrica, Departamento de Pediatria, ${ }^{2}$ Divisão de Reumatologia, ${ }^{3}$ Divisão de Urologia, ${ }^{4}$ Divisão de Radiologia, Faculdade de Medicina, Universidade de São Paulo, São Paulo, SP, Brasil

Correspondence to: C.A.A. Silva, Rua Araioses, 152/81, 05442-010 São Paulo, SP, Brasil

Fax: +55-11-3069-8503. E-mail: clovis.silva@icr.usp.br

The objective of the present study was to identify sperm abnormalities in young male patients with juvenile dermatomyositis (JDM). In 2005, 18 male JDM patients, diagnosed according to the criteria of Bohan and Peter, were followed at the Pediatric Rheumatology Unit and Rheumatology Division, of our Institution. Of the 18 males, 11 were pre-pubertal and 7 were postpubertal. Two of 7 post-pubertal JDM male patients were excluded: one for orchidopexy for cryptorchidism and the other for testicular ectopia in the left testis. The remaining 5 post-pubertal JDM patients were prospectively evaluated on the basis of two semen analyses, according to the World Health Organization (WHO), urologic evaluation, testicular Doppler ultrasound hormone profile. The data of the JDM patients were compared with those of 5 age-matched healthy controls. The median age 18 , was similar in JDM patients and controls. All JDM patients had teratozoospermia (abnormal sperm morphology), as did 4 $(80 \%)$ of the controls. One of JDM patients had previous oligoasthenoteratozoospermia treated with intravenous cyclophosphamide with normalization of the number and concentration of the sperm after 5 years. All sperm parameters (sperm concentration, total sperm count and total motile sperm count by WHO, and sperm morphology by Kruger strict criteria), testicular volumes by Prader orchidometer and ultrasound, and hormones were similar in JDM patients compared with controls. The frequency of antisperm antibodies was similar in both groups. All JDM patients had minor sperm abnormalities in the head, midpiece, and/or tail of spermatozoids. Serial semen analyses in larger study populations are necessary to identify the extent and duration of sperm abnormalities in male patients with idiopathic inflammatory myopathies.

Key words: Juvenile dermatomyositis; Gonad; Sperm; Hormone; Male post-puberty; Idiopathic inflammatory myopathy

Research supported by FAPESP (\#2005/56482-7) and CNPq (\#302469/2005-2).

Received June 6, 2008. Accepted November 10, 2008

\section{Introduction}

Juvenile dermatomyositis (JDM) is a rare multisystemic disease affecting 2 to 4 children per million, particularly males, characterized by vasculitis that affects the skin and muscles (1). Survival and prognosis for patients with JDM have improved in the last decades. JDM patients are living longer and clinical attention has begun to shift toward improving their quality of life. Upon becoming adolescents and young adults, male JDM patients are often concerned about their future fertility. Evaluation of young male gonadal function requires a complete urological examination in order to determine minor or major sperm alterations due to age, genital system abnormalities [e.g., hydrocele, hypospadia, cryptorchidism, testicular infection (e.g., mumps), testicular cancer], influence of drugs especially intravenous cyclophosphamide (IVCYC), and antisperm antibodies (2). Of note, we recently identified sperm abnormalities in young $(2,3)$ and adult male systemic lupus erythematosus (SLE) patients associated with IVCYC treatment (4). 
In JDM, disease activity may be an additional contributing factor for possible testicular damage, supported by the report of one case of testicular vasculitis in a 7-year-old boy (5). However, there is no systematic study assessing the overall gonad function in young male JDM patients and its relevance for sperm abnormalities.

The objective of this study was to perform a global testicular assessment in post-pubertal male JDM patients and a matched control group.

\section{Subjects and Methods}

In 2005, 18 male JDM patients, diagnosed according to Bohan and Peter criteria (6), were followed at the Pediatric Rheumatology Unit and at the Rheumatology Division, of our institution. Eleven were pre-pubertal and 7 were postpubertal. Exclusion criteria were hydrocele, hypospadia, cryptorchidism, testicular infection (e.g., mumps), testicular cancer, orchitis, testicular vasculitis, urethral impairment, previous history of any scrotal or inguinal surgery (e.g., varicocelectomy, vasectomy, hernia repair), diabetes mellitus, previous or current history of alcohol or tobacco use, and refusal to collect sperm sample or incomplete evaluation. Two of 7 post-pubertal JDM male patients were excluded due to orchidopexy for cryptorchidism in one and testicular ectopia in the left testis in the other. The remaining 5 JDM patients were compared with a control group of 5 age-matched healthy male adolescents regularly followed at the Adolescent Unit. The Local Ethics Committee approved this study, and written informed consent was obtained from all participants or from a parent, if necessary.

An extensive clinical evaluation by the same rheumatologist was followed by a careful chart review including previous clinical, laboratory, and treatment-related data. JDM manifestations were defined as cutaneous lesions (heliotrope, Gottron's papules, vasculitis, calcinosis, ulcerative skin or malar rash), articular involvement (arthralgia or nonerosive arthritis), muscular involvement (muscle weakness, muscle edema), cardiopulmonary disease (serositis, myocarditis, restrictive lung disease, and pulmonary hypertension), and intestinal involvement (dysphagia or vasculitis). JDM disease activity and damage were measured in all patients using the following tests: Childhood Health Assessment Questionnaire (CHAQ) (7), Manual Muscle Testing (8), Childhood Myositis Assessment Scale (CMAS) (9), and Disease Activity Score (DAS) (10). Physician and patient assessments of global disease activity and damage were rated on a $10-\mathrm{cm}$ visual analog scale (VAS) (11) and physical functioning was assessed by the Medical Outcomes Study 36 Item Short-Form Health
Survey (PF-SF-36) (12). Data about the period of therapy (pre-pubertal or post-pubertal) were collected as well as current dosage and cumulative doses of drugs (prednisone, chloroquine diphosphate, methotrexate, azathioprine, cyclosporine, colchicine, and IVCYC). The time and duration of IVCYC therapy were also determined.

A systematic clinical examination of the genitalia was performed on patients and controls by the same expert urologist and included evaluation of testicles, epididymis, vas deferens, scrotum, and penis. The secondary sexual characteristics were evaluated according to Tanner's pattern criteria of pubertal changes (13). Testicular volume was measured using Prader orchidometry, which consists of 12 ellipsoid models graded from 1 to $25 \mathrm{~mL}$ (1-6, 8, 10, $12,15,20$, and 25) (14). The patients were examined in a warm room with temperature not $<22^{\circ} \mathrm{C}$, in both the standing and supine positions, and with and without Valsalva maneuver. Varicocele was graded according to the following criteria: grade I (small) = palpable only with concurrent Valsalva maneuver; grade II (medium) = palpable with patient standing, and grade III (large) = visible through scrotal skin, palpable with patient standing (15). Ultrasound was performed on all JDM patients and control subjects by an expert sonographer who used a $14-\mathrm{MHz}$ sector scanner (Logic 9; GE, USA) and who was not aware of the results of the semen analysis. Testes were scanned in axial and longitudinal planes, and at least 2 measurements of length, width, and thickness were obtained. The largest measurement in each dimension was recorded and used to calculate the testicular volume according to the formula for an ellipsoid (length $x$ width $x$ thickness $x$ 0.52). The mean \pm SD value for normal male post-pubertal adolescents and adults is $15 \pm 8 \mathrm{~mL}$ (15).

Semen analysis was performed according to the guidelines of the World Health Organization (WHO) (16) by 2 expert medical technologists who were not aware of disease diagnosis. Within 1 month after acceptance into the study, all JDM patients and controls were asked to provide, after 48-72 h of sexual abstinence, 2 semen samples, which were collected by masturbation in the laboratory collection room and processed within $1 \mathrm{~h}$ of liquefaction. Semen specimens were liquefied at $37^{\circ} \mathrm{C}$ for $30 \mathrm{~min}$, and sperm concentration and motility were measured manually with a microscope fitted with a $20-X$ positive phase-contrast objective and an overall magnification of 200X. Microscopic images were transferred through a video system to a computer-assisted semen analyzer attached to the optical microscope and were digitized according to a software program. The specimens were analyzed by manual hand count as well as by a computer-assisted semen analysis system under 400X magnification (HTM-2030 motility ana- 
lyzer, Hamilton Thorne Research, USA). Each slide was scanned to estimate the number of spermatozoa per field equivalent to $1 \mathrm{~mL}$ to obtain an approximate sperm concentration reported in millions of spermatozoa per milliliter semen. Sperm motility was determined by analysis of at least 5 microscopic fields in a systematic manner to classify 200 spermatozoa. The motility of each spermatozoon was graded "a" (rapid progressive motility) and "b" (slow or sluggish progressive motility). Sperm morphology included evaluation of sperm head, neck, midpiece, and tail by 2 medical technologists who were not aware of disease diagnosis. A patient was considered to have azoospermia when no spermatozoa were found in the ejaculate (16). Oligozoospermia was defined as a sperm concentration $<20$ million/mL. Asthenozoospermia was defined as normal sperm motility ("a" + "b") in $<50 \%$ of spermatozoa. Teratozoospermia was defined as normal sperm morphology in $<30 \%$ of spermatozoa by WHO criteria, and oligoasthenoteratozoospermia was defined as an alteration in all 3 variables. Sperm morphology was additionally evaluated according to Kruger strict criteria, in which $<14 \%$ normal morphology is associated with subfertility (17).

The presence of antisperm antibodies was determined by direct Immunobead test using Immunobead rabbit antihuman $\lg (\lg A, \lg G$, and $\lg M)$ kits (Irvine Scientific, USA). The direct Immunobead binding test detects antibodies that bind to the sperm cell surface (sperm head, midpiece, and/or tail). At least $50 \%$ of the motile spermatozoa ("a" + "b") must be coated with Immunobeads before the test results are considered to be clinically significant (16). Quality control was carried out as recommended by the manufacturer (Irvine Scientific, USA). A negative control should have a score of $<10 \%$ bead attachment, and a positive control should have a score $>20 \%$ bead attachment.

Hormone determinations were performed at study entry. Follicle-stimulating hormone (FSH), luteinizing hormone (LH), prolactin, total testosterone, triiodothyronine (T3), tetraiodothyronine (T4), free tetraiodothyronine (free T4), and thyrotropin (TSH) were evaluated by fluoroimmunoassay using DELFIA time-resolved fluoroimmunoassay kits (Wallac, Finland). Intra- and interassay coefficients of variation were limited to 3.5 and $2.1 \%$, respectively.

Data were compared by the $t$-test or by the MannWhitney U-test for continuous variables to evaluate differences between the JDM patients and the control group. $P$ values less than 0.05 were considered to be significant.

\section{Results}

The clinical features, laboratory exams, previous and current therapy in 5 male JDM patients are reported in
Table 1. All patients had normal CMAS, DAS, CHAQ, and PF-SF-36 scores. Disease activity was observed in 2 JDM patients: patients 2 and 3 had higher scores of DAS, VAS as reported by the patient, and VAS as reported by the physician ( 2 and 5, 3 and 5, 4 and 5, respectively). All patients received drugs after puberty with the exception of 2 who were treated with steroid, chloroquine diphosphate and colchicine therapies in the pre-pubertal period.

The medians of current age and age of first ejaculation were similar in JDM patients and controls [18 years (range 16-22) vs 18 years (range 16-22), $P=1.0$, and 13 years (range 13-14) vs 13 years (range 12-15), $P=0.785$, respectively]. All JDM patients and controls were classified as P5 and G5, respectively, according to sex maturity states by Tanner's pattern criteria of pubertal changes (13). The median values of the testicular volumes in both testes according to Prader orchidometry were similar in JDM patients and controls (20 vs $20 \mathrm{~mL}$ in the right testicle, and 20 vs $20 \mathrm{~mL}$ in the left testicle, respectively). The medians of the testicular volumes in both testes according to ultrasound were homogeneous in JDM patients and controls (10.6 vs $11.7 \mathrm{~mL}$ in the right testicle, and $10.2 \mathrm{vs}$ $10.5 \mathrm{~mL}$ in the left testicle, respectively). In addition, 2 JDM patients and 2 controls had varicocele grade I (40 vs $40 \%$ ). All JDM patients and controls had normal testicular volumes by ultrasound and Prader orchidometry.

All 5 JDM patients (100\%) had teratozoospermia (abnormal sperm morphology in the head, midpiece, and/or tail of spermatozoids), as did 4 controls $(80 \%, P=1.0)$. Remarkably, one of the JDM patients (case 4) had previous oligoasthenoteratozoospermia (low sperm concentration, motility and morphology) with IVCYC treatment [14 monthly infusions with $500 \mathrm{mg} / \mathrm{m}^{2}$ and a cumulative dose of $6.6 \mathrm{~g}(150 \mathrm{mg} / \mathrm{kg})$ ] with normalization of the number and concentration of sperm after 5 years. No patient had azoospermia.

The mean of 2 sperm analyses in JDM patients was compared with controls. No significant differences were found between JDM patients and controls in median sexual abstinence and sperm volume (3.5 vs 3.4 days, $P=0.564$, and 2.8 vs $2.4 \mathrm{~mL}, \mathrm{P}=0.465$, respectively). Likewise, no significant difference was observed in median sperm $\mathrm{pH}$ (7.7 vs 7.5; $\mathrm{P}=0.059$ ). The microscopic investigation showed that JDM patients had median sperm concentrations $\left(50 \times 10^{6} / \mathrm{mL}\right.$ vs $\left.60.5 \times 10^{6} / \mathrm{mL} ; \mathrm{P}=0.5\right)$, total sperm count $\left(212 \times 10^{6} / \mathrm{mL}\right.$ vs $\left.120 \times 10^{6} / \mathrm{mL} ; \mathrm{P}=0.5\right)$ and total motile sperm count $\left(74 \times 10^{6} / \mathrm{mL}\right.$ vs $\left.70 \times 106 / \mathrm{mL} ; \mathrm{P}=0.893\right)$ similar to controls. Assessment of sperm morphology by Kruger strict criteria demonstrated similar median normal sperm forms in JDM patients and controls (5.0 vs 4.5\%; $\mathrm{P}$ $=0.715$ ) as also observed by the WHO criteria (19 vs $18 \%$; 
Table 1. Demographic data, clinical features, laboratory exams, previous and current therapy in 5 male juvenile dermatomyositis patients.

\begin{tabular}{|c|c|c|c|c|c|}
\hline Variables & Patient 1 & Patient 2 & Patient 3 & Patient 4 & Patient 5 \\
\hline Age at disease onset & 9 years & 3 years & 13 years & 15 years & 6 years \\
\hline $\begin{array}{l}\text { Disease duration at } \\
\text { study entry }\end{array}$ & 8 years & 13 years & 7 years & 7 years & 12 years \\
\hline $\begin{array}{l}\text { JDM clinical } \\
\text { manifestation and } \\
\text { laboratory exams }\end{array}$ & $\begin{array}{l}\text { Muscle weakness, } \\
\text { heliotrope, } \\
\text { Gottron's papules, } \\
\text { 个muscle enzymes }\end{array}$ & $\begin{array}{l}\text { Severe muscle weakness, } \\
\text { heliotrope, } \\
\text { Gottron’s papules, } \\
\text { calcinosis, } \\
\text { 个muscle enzymes }\end{array}$ & $\begin{array}{l}\text { Severe muscle } \\
\text { weakness, heliotrope, } \\
\text { Gottron's papules, } \\
\text { pyrexia, weight loss, } \\
\text { ulcerative skin, } \\
\text { calcinosis, } \\
\text { 个muscle enzymes }\end{array}$ & $\begin{array}{l}\text { Severe muscle } \\
\text { weakness, heliotrope, } \\
\text { Gottron's papules, } \\
\text { dysphagia, pyrexia, } \\
\text { duodenal vasculitis, } \\
\text { 个muscle enzymes }\end{array}$ & $\begin{array}{l}\text { Muscle weakness, } \\
\text { pyrexia, heliotrope, } \\
\text { Gottron's papules, } \\
\text { weight loss, fatigue, } \\
\text { 个muscle enzymes }\end{array}$ \\
\hline
\end{tabular}

\begin{tabular}{|c|c|c|c|c|c|}
\hline \multicolumn{6}{|l|}{ Cumulative dose (g) } \\
\hline PRED & 8.68 & 31.4 & 9.4 & 25.6 & 16.2 \\
\hline IVCYC & 0 & 0 & 0 & 6.6 & 0 \\
\hline MTX & 0 & 0.16 & 0.465 & 0 & 0 \\
\hline$A Z A$ & 0 & 0 & 0 & 71.2 & 0 \\
\hline$C D$ & 0 & 72 & 487.5 & 30 & 0 \\
\hline COL & 0 & 0 & 0 & 0 & 0.78 \\
\hline \multirow[t]{3}{*}{ Current medication } & - & PRED 10 mg/day & PRED 30 mg/day & - & - \\
\hline & & & MTX 15 mg/week & - & - \\
\hline & & & CD 50 mg/day & - & - \\
\hline
\end{tabular}

$\mathrm{PRED}=$ prednisone; IVCYC = intravenous cyclophosphamide; $\mathrm{MTX}=$ methotrexate; $\mathrm{AZA}=$ azathioprine; $\mathrm{CD}=$ chloroquine diphosphate; $\mathrm{COL}=$ colchicine.

$P=0.893)$. Of note, one of the JDM patients with cutaneous active disease had anti-sperm antibodies (>20\%) in the spermatozoon mid-piece and none of the controls had the presence of these antibodies (1.0).

All JDM patients and controls had normal hormone profiles. No significant differences were found between the medians in the 2 groups: FSH (3.3 vs $3.6 \mathrm{IU} / \mathrm{L} ; \mathrm{P}=0.686)$, $\mathrm{LH}$ (3.7 vs $3.7 \mathrm{IU} / \mathrm{L} ; \mathrm{P}=0.893)$, prolactin (7.1 vs $6.4 \mathrm{ng} / \mathrm{mL}$; $\mathrm{P}=0.500)$, total testosterone (394 vs $677 \mathrm{ng} / \mathrm{dL} ; \mathrm{P}=$ $0.138)$, T4 (8.9 vs $9.3 \mu \mathrm{g} / \mathrm{dL} ; \mathrm{P}=0.893)$, free T4 (1.3 vs 1.2 $\mathrm{ng} / \mathrm{dL} ; \mathrm{P}=0.684)$, and TSH (2.1 vs $2.2 \mu \mathrm{U} / \mathrm{mL} ; \mathrm{P}=0.686)$. The median T3 levels were significantly higher in JDM patients compared with controls (152 vs $140 \mathrm{ng} / \mathrm{dL} ; \mathrm{P}=$ 0.043 ); however, these levels are normal for these hormones.

\section{Discussion}

This is the first systematic evaluation that has specifically addressed sperm abnormalities in post-pubertal JDM. Despite a very small sample size, we evaluated a control population and demonstrated minor semen alterations in both groups. Remarkably, one of our patients had previously severe sperm abnormalities during IVCYC use.

An advantage of our study was in using established worldwide definitions for semen alterations. Teratozoospermia was observed in $100 \%$ of JDM patients and $80 \%$ of controls. These patients and controls could be classified as subfertile concerning the cutoff values for normality in 2 conventional techniques of semen morphology. Moreover, Mori et al. (18) studied 127 healthy adolescents (age, 1420 years) in the city of São Paulo and observed teratozoospermia in $82 \%$ of the adolescents who were classified as P5 and G5 according to Tanner's pattern criteria of pubertal changes (13). Likewise, an Italian study evaluated 25 healthy adolescents who were 14 to 18 years old and observed a prevalence of abnormal forms of spermatozoon in $56 \%$ (19). Remarkably, we recently studied gonad evaluation in 35 male adolescents and adult SLE patients and 35 controls paired by age. Teratozoospermia was observed in $100 \%$ of SLE patients vs $85 \%$ of controls (7), similar to that observed here for our young male JDM patients. All adult controls with teratozoospermia fathered children. Furthermore, regional differences in se- 
men morphology, possibly associated with environmental exposures or lifestyle, may explain the teratozoospermia, as has been reported in the United States (20).

Testicular volume assessment is of utmost importance when evaluating gonadal function due to the fact that seminiferous tubules represent $95 \%$ of the testis volume. It is very important to perform a testicular ultrasound to rule out varicocele, even when testicular volume is normal (15). In our study, testicular volume was normal in all patients and controls, grade I varicocele was observed in one patient and in one control with normal testis volume. Although varicocele is believed to be one of the most common causes of male subfertility, grade I varicocele has no detectable sperm abnormalities and dysfunction of the hypothalamic-pituitary-testicular axis (2). FSH is the major marker of seminiferous epithelium function, and high levels suggest definitive testicular damage (2). All our patients had normal hormone levels reinforcing that male JDM patients had normal gonadal function.

A possible role of antisperm antibodies as a marker for

\section{References}

1. Sallum AM, Kiss MH, Sachetti $S$, Resende MB, Moutinho $\mathrm{KC}$, Carvalho MS, et al. Juvenile dermatomyositis: clinical, laboratorial, histological, therapeutical and evolutive parameters of 35 patients. Arq Neuropsiquiatr 2002; 60: 889-899.

2. Silva CA, Brunner HI. Gonadal functioning and preservation of reproductive fitness with juvenile systemic lupus erythematosus. Lupus 2007; 16: 593-599.

3. Silva CA, Hallak J, Pasqualotto FF, Barba MF, Saito MI, Kiss $\mathrm{MH}$. Gonadal function in male adolescents and young males with juvenile onset systemic lupus erythematosus. $J$ Rheumatol 2002; 29: 2000-2005.

4. Soares PM, Borba EF, Bonfa E, Hallak J, Correa AL, Silva CA. Gonad evaluation in male systemic lupus erythematosus. Arthritis Rheum 2007; 56: 2352-2361.

5. Jalleh RP, Swift RI, Sundaresan M, Toma A, Wood CB. Necrotising testicular vasculitis associated with dermatomyositis. Br J Urol 1990; 66: 660.

6. Bohan A, Peter JB. Polymyositis and dermatomyositis (first of two parts). N Engl J Med 1975; 292: 344-347.

7. Machado CS, Ruperto N, Silva CH, Ferriani VP, Roscoe I, Campos LM, et al. The Brazilian version of the Childhood Health Assessment Questionnaire (CHAQ) and the Child Health Questionnaire (CHQ). Clin Exp Rheumatol 2001; 19: S25-S29.

8. Legg AT, Merril JB. Physical therapy in infantile paralysis. In: Legg AT, Merril JB (Editors), Principles and practice of physical therapy. Hagerstown: Mock Pemberton and Coulter; 1932.

9. Lovell DJ, Lindsley CB, Rennebohm RM, Ballinger SH, Bowyer SL, Giannini EH, et al. Development of validated gonadal dysfunction was seen in 1 patient with active JDM. Soares et al. (4) showed a higher frequency of these antibodies in Brazilian SLE patients with teratozoospermia (56\%) versus SLE with azoospermia or oligoasthenoteratozoospermia (24\%), with the same methodology as the present study.

The testis is susceptible to the toxic effects of IVCYC therapy, which might produce long-lasting or persistent damage to primordial sperm cells, leading to oligoasthenoteratozoospermia in patients with juvenile $\operatorname{SLE}(2,3)$ or SLE (4). Indeed, sperm cryopreservation should be discussed as a routine part of therapeutic management to preserve fertility in patients who will receive IVCYC (2).

We have shown here that JDM patients had minor sperm abnormalities. Serial semen analyses in larger study populations are necessary to determine the degree and duration of sperm abnormalities in male patients with idiopathic inflammatory myopathies, particularly associated with immunosuppressive drugs.

disease activity and damage indices for the juvenile idiopathic inflammatory myopathies. II. The Childhood Myositis Assessment Scale (CMAS): a quantitative tool for the evaluation of muscle function. The Juvenile Dermatomyositis Disease Activity Collaborative Study Group. Arthritis Rheum 1999; 42: 2213-2219.

10. Bode RK, Klein-Gitelman MS, Miller ML, Lechman TS, Pachman LM. Disease activity score for children with juvenile dermatomyositis: reliability and validity evidence. Arthritis Rheum 2003; 49: 7-15.

11. Rider LG, Feldman BM, Perez MD, Rennebohm RM, Lindsley CB, Zemel LS, et al. Development of validated disease activity and damage indices for the juvenile idiopathic inflammatory myopathies: I. Physician, parent, and patient global assessments. Juvenile Dermatomyositis Disease Activity Collaborative Study Group. Arthritis Rheum 1997; 40: 1976-1983.

12. da Mota Falcao D, Ciconelli RM, Ferraz MB. Translation and cultural adaptation of quality of life questionnaires: an evaluation of methodology. J Rheumatol 2003; 30: 379-385.

13. Tanner JM. Growth at adolescence. 2nd edn. Oxford: Blackwell Scientific Publications; 1962.

14. Prader A. Testicular size: assessment and clinical importance. Triangle 1966; 7: 240-243.

15. Atkinson GO Jr, Patrick LE, Ball TI Jr, Stephenson CA, Broecker BH, Woodard JR. The normal and abnormal scrotum in children: evaluation with color Doppler sonography. AJR Am J Roentgenol 1992; 158: 613-617.

16. Rowe PJ, Comhaire FH, Hargreave TB, Mahmoud AM. World Health Organization (WHO) manual for the standard- 
ized investigation, diagnosis and management of the infertile male. 1st edn. Cambridge: Cambridge University Press; 2000.

17. Kruger TF, Acosta AA, Simmons KF, Swanson RJ, Matta $\mathrm{JF}$, Oehninger S. Predictive value of abnormal sperm morphology in in vitro fertilization. Fertil Steril 1988; 49: 112117.

18. Mori MM, Cedenho AP, Koifman S, Srougi M. Sperm char- acteristics in a sample of healthy adolescents in São Paulo, Brazil. Cad Saude Publica 2002; 18: 525-530.

19. Paris E, Menchetti A, De Lazzaro L, Marrocco M, Nuzzo C, Radicioni A. The spermiogram in adolescence. Minerva Pediatr 1998; 50: 303.

20. Swan SH, Brazil C, Drobnis EZ, Liu F, Kruse RL, Hatch M, et al. Geographic differences in semen quality of fertile U.S. males. Environ Health Perspect 2003; 111: 414-420. 